


\title{
COMPOSIÇÃO FLORÍSTICA E FITOSSOCIOLÓGICA DO CERRADO SENSU STRICTO NO PARQUE ESTADUAL DA LAPA GRANDE, MONTES CLAROS, MG
}

\author{
Kelly Marianne Guimarães Pereira1; Christian Dias Cabacinha2; Lisandra Maria Alves \\ Matos; ; Inkamauta Cerda Valeda Plazas ${ }^{4}$; \\ ${ }^{1}$ Graduanda pela Universidade Federal de Minas Gerais, Montes Claros, Minas \\ Gerais, Brasil. E-mail: kellyguimaraes10@gmail.com \\ ${ }^{2}$ Professor Doutor da Universidade Federal de Minas Gerais \\ ${ }^{3}$ Graduanda da Universidade Federal de Minas Gerais \\ ${ }^{4}$ Graduando da Universidade Estadual do Sudoeste da Bahia
}

Recebido em: 03/10/2016 - Aprovado em: 21/11/2016 - Publicado em: 05/12/2016 DOI: 10.18677/EnciBio_2016B_026

\begin{abstract}
RESUMO
O Parque Estadual da Lapa Grande (PELG), localizado no norte de Minas Gerais está inserido no Cerrado que possui uma diversidade única. Com o objetivo de caracterizar a comunidade vegetal, fez-se um estudo fitossociológico e florístico do cerrado sensu stricto e selecionou espécies mais representativas da vegetação. As parcelas possuíam dimensões de $400 \mathrm{~m}^{2}(20 \times 20 \mathrm{~m})$ com método de inclusão de Diâmetro a Altura do Solo (DAS) acima de $3 \mathrm{~cm}$, sendo coletados o diâmetro, altura total e o material botânico dos indivíduos. Tais dados foram processados no FITOPAC, obtendo os parâmetros fitossociológicos e florísticos. As espécies que apresentaram maiores valores nos quesitos de abundância, distribuição espacial, densidade, frequência, e IVI foram classificadas em relação à síndrome de dispersão e caracterizadas quanto a sua fenologia. As famílias Fabaceae, Vochysiaceae, Myrtaceae e Malvaceae representaram $51,24 \%$ de todos os indivíduos amostrados. A área apresentou um índice de Shannon com valor de 4,12 nats.indivíduo ${ }^{-1}$, considerado alto quando comparado a outros estudos. As espécies mais representativas foram 22 , com um comportamento de frutificação aumentando entre os meses de agosto e fevereiro e floração com um aumento a partir de julho e queda em setembro. Em relação à dispersão, as espécies apresentaram em sua maioria comportamento de anemocórica.
\end{abstract}

PALAVRAS-CHAVE: Caracterização; Diversidade; Conservação.

\section{FLORISTIC COMPOSITION AND PHYTOSOCIOLOGICAL OF THE CERRADO SENSU STRICTO AT THE LAPA GRANDE STATE PARK, MONTES CLAROS, MG}

\begin{abstract}
The Lapa Grande State Park (PELG) is situated in the north of Minas Gerais, it is in the Cerrado that has a unique diversity. A phytosociological and floristic study of the cerrado sensu stricto was made with the purpose of characterize the plant community and selected of the most representative species of vegetation. The plots had $400 \mathrm{~m}^{2}(20 \times 20 \mathrm{~m})$ and it was measured all trees with the diameter of tree base above $3 \mathrm{~cm}$. It was collected the diameter, total height and the individuals botanical material. The data were processed using the FITOPAC software getting as result the
\end{abstract}


phytosociological and floristic parameters. The species that showed higher values in the categories of abundance, spatial distribution, density, frequency, and IVI were classified according to the dispersal syndrome and characterized by its phenology. The Fabaceae, Vochysiaceae, Myrtaceae and Malvaceae families represented $51.24 \%$ of all sampled individuals. The Shannon index to the area was 4.12 nats.individuals ${ }^{-1}$, being considered as high index when compared to other studies. In general, 22 species increased fruiting between August and February and flowering increased from July and decreased in September. In this respect, the species showed dispersion mostly anemocoric.

KEYWORDS: Characterization; diversity; conservation.

\section{INTRODUÇ̃̃O}

O Parque Estadual da Lapa Grande (PELG) está situado na cidade de Montes Claros (norte de Minas Gerais), sendo instituído pelo Decreto no 44.204 no dia 10 de janeiro de 2006. O principal objetivo da sua criação foi proteger e conservar o complexo de grutas, mananciais, flora e fauna, de grande diversidade presente no local, que assume grande importância na região de Montes Claros, contendo os principais mananciais de abastecimento, já que cerca de $30 \%$ da água da região é oriunda do parque (MINAS GERAIS, 2006).

A Unidade tem apresentado extensas áreas degradadas, com predominância de gramíneas, em grande parte exótica, já que a mesma foi formada por antigas fazendas que utilizavam suas terras para a criação de animais, e também pelo fato de que muitas áreas foram atingidas por queimadas. Em novembro de 2015 um incêndio criminoso destruiu uma área considerável na Unidade, intensificando a importância da conservação dos seus recursos.

O PELG está inserido em área do bioma Cerrado que é considerado um dos hotspots mundiais, representando $25 \%$ do território brasileiro. Apesar da sua biodiversidade comprovada, o bioma vem sendo ameaçado com o desmatamento, ocupação humana, expansão agrícola e florestal, entre outras atividades antrópicas, que vem causando a fragmentação da vegetação. Além disso, existem poucas áreas protegidas que dificulta a conservação da quantidade de espécies endêmicas e desconhecidas, o que tem tornado os estudos no Cerrado cada vez mais importante (BRASIL, 2007).

Uma das fitosionomias do Cerrado é o sensu stricto que é caracterizada por possuir uma vegetação com cascas grossas, gemas apicais com uma proteção de tricomas, folhas com aspecto rígido, apresentando o xeromorfismo (adaptação a situações de déficit hídrico) (RIBEIRO \& WALTER, 1998). Sendo assim, para conhecer o comportamento da vegetação de forma ampla, os levantamentos fitossociológicos são fundamentais para o fornecimento de dados e informações indispensáveis visando à padronização de características florísticas e fisionômicas, o que possibilitará a conservação e preservação de ecossistemas (OLIVEIRA \& AMARAL, 2004).

Por meio desses levantamentos é possível distinguir diferentes espécies, que são imprescindíveis para o uso sustentável do Cerrado, pois este abriga uma diversidade comprovadamente única, além disso, uma grande riqueza florística e heterogeneidade de ambientes (BRASIL, 2011). A amostragem também é um fator a ser analisado para realização de caracterização da vegetação, pois a representação da diversidade de forma satisfatória está inteiramente ligada a esse fator (FERREIRA et al., 2016). 
Neste contexto, este trabalho teve como objetivo caracterizar a comunidade vegetal de Cerrado sensu stricto do interior do Parque Estadual da Lapa Grande quanto à sua estrutura fitossociológica e florística bem como listar as espécies mais representativas da área.

\section{MATERIAL E MÉTODOS}

\section{Caracterização e localização do Parque Estadual da Lapa Grande}

O estudo foi realizado no Parque Estadual da Lapa Grande (PELG), município de Montes Claros, no estado de Minas Gerais que está localizado nas coordenadas: 160'19,6" e 1646'34,0" S e 4354'07,4" a 4400' 15,3" W (OLIVEIRA et al., 2015) (Figura 1).

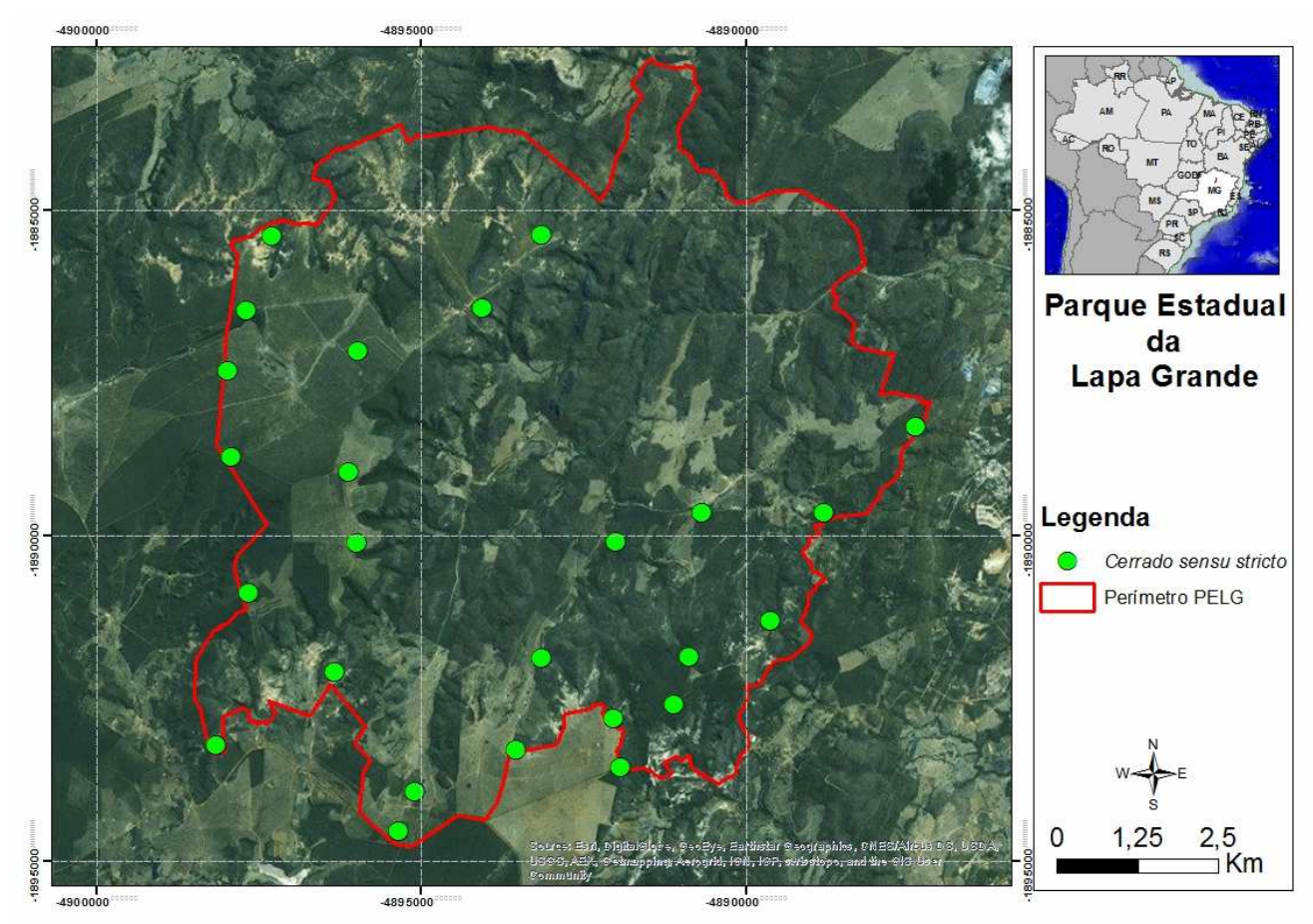

FIGURA 1 - Mapa do PELG com a localização das parcelas amostradas no Cerrado sensu stricto.

Fonte: Autores

A área da unidade correspondia a 7000 ha e a partir de 2014 por meio do Decreto 46.692 de 29 de dezembro de 2014 a área do parque foi ampliada para mais de 15 mil hectares (MINAS GERAIS, 2015). A unidade possui uma vegetação predominante de Cerrado sensu stricto, Floresta Estacional Decidual (Mata Seca) e Matas Ciliares (IBGE, 2012), abrigando também 62 cavidades, com presença de uma rica fauna (MINAS GERAIS, 2015).

\section{Coleta de Dados}

Os dados das áreas de cerrado sensu stricto foram coletados por meio de demarcação de pontos pelos trechos de vegetação. Esses trechos eram identificados e indicados através de mapas temáticos. O método utilizado foi o de parcelas de área fixa de $400 \mathrm{~m}^{2}(20 \times 20 \mathrm{~m})$. Foram aleatorizadas 25 unidades amostrais, totalizando um hectare (MORO \& MARTINS, 2011). A coleta foi realizada 
durante os anos de 2013 e 2014, e as parcelas foram delimitadas com piquetes de 1 metro.

O método de inclusão utilizado foi a amostragem de indivíduos lenhosos vivos, com Diâmetro a Altura do Solo (DAS) maior ou igual a $3 \mathrm{~cm}$, pois esse método proporciona uma melhor caracterização da vegetação. As árvores que atendiam essa exigência tiveram o diâmetro e a altura total, medidos, além da coleta de material botânico para herborização e posterior identificação. A última variável foi estimada com uso de vara graduada. Os indivíduos mensurados foram marcados com placas de alumínio (MORO \& MARTINS, 2011).

O material botânico foi identificado inicialmente em campo, por meio do nome vulgar, sendo identificados em definitivo com consulta em literatura especializada (SILVA-JUNIOR, 2005; SILVA-JUNIOR \& PEREIRA, 2009), herbários virtuais (FLORA DO BRASIL, 2016; JBRJ, 2016).

\section{Processamento dos dados}

Os dados obtidos foram organizados em planilhas eletrônicas do software Excel, com o número da parcela, o nome científico, DAP e altura. Essas planilhas foram processadas no software FITOPAC, obtendo os parâmetros fitossociológicos e florísticos da vegetação, como, riqueza, densidade relativa, frequência relativa, dominância relativa, índice de valor de importância (MULLER-DOMBOIS \& ELLENBERG, 1974), posição sociológica relativa e valor de importância ampliado (SOUZA \& SOARES, 2013). Além disso, foi calculada a diversidade pelo índice de Shannou e equabilidade de Pielou (MAGURRAN, 2011).

As espécies que apresentaram maiores valores de abundância, densidade relativa, frequência relativa, IVI e estava presente em um terço das unidades amostrais foram as mais representativas, com maior taxa de ocupação e maior número de indivíduos no levantamento do cerrado sensu stricto. Posteriormente essas espécies foram caracterizadas de acordo com a fenologia e classificadas pela síndrome de dispersão em: anemocóricas, autocóricas e zoocóricas (VAN DER PIJL, 1982).

\section{RESULTADOS E DISCUSSÃO}

$\mathrm{Na}$ área amostrada foram registrados 3059 indivíduos, sendo 3046 identificados, pertencentes a 171 espécies, distribuídas 46 famílias. As famílias que apresentaram o maior número de espécies foram Fabaceae e Myrtaceae, com 28 e 18 espécies respectivamente, pois o Parque possui muitas áreas de transição de Cerrado sensu stricto para a Floresta Estacional Semidecidual (Mata Seca). Em ordem decrescente seguiram as famílias: Vochysiaceae (9 espécies), Asteraceae e Apocynaceae (8), Anacardiaceae e Bignoniaceae (7), Malvaceae, Annonaceae, Combretaceae, Malpighiaceae, Nyctaginaceae (5), Rubiaceae e Erythroxylaceae (4), Melastomataceae e Salicaceae (3), Sapindaceaae, Sapotaceae, Dilleniaceae, Meliaceae, Ochnaceae, Polygonaceae, Calophyllaceae e Verbenaceae (2). As outras famílias apresentaram apenas uma espécie cada. Dos 13 indivíduos não identificados, dois foram classificados até o nível de família e 11 as famílias não foram identificadas.

JACOBI et al. (2008) salientam que as famílias mais representativas por número de espécies e também pela sua abundância de indivíduos devem ser consideradas para o manejo da vegetação, pois esses índices demonstram que o táxon conseguiu adaptar-se bem no ambiente. As quatro famílias com maior 
porcentagem de indivíduos foram as seguintes: Fabaceae, Vochysiaceae, Myrtaceae e Malvaceae, apresentando um total de $51,24 \%$ dos indivíduos, com a família Fabaceae, ocupando a primeira posição com 23,54\%, sendo seguidas por Vochysiaceae (12,68\%), Myrtaceae (7,49\%) e Malvaceae $(7,36 \%)$.

A família Fabaceae tem sido normalmente encontrada com maior representatividade em áreas de cerrado sensu stricto (ALMEIDA et al., 2014; FINGER; FINGER, 2015). Outros estudos realizados no norte de Minas mostram que famílias citadas foram comumente encontradas com maior número de espécies, exceto a família Malvaceae (LIMA et al., 2012; SOARES \& NUNES, 2013).

As dez espécies que destacaram com maiores Índice de Valor de Importância (IVI) totalizaram $34,1 \%$ dos indivíduos, e foram em ordem decrescente: Qualea grandiflora Mart. (6,98\%), Eriotheca pubescens (Mart. \& Zucc.) Schott \& Endl. (5,24\%), Magonia pubescens A.St.-Hil. (3,48\%), Machaerium opacum Vogel $(3,13 \%)$, Hymenaea stigonocarpa Mart. ex Hayne (3,09\%), Copaifera langsdorffii Desf. (3,00\%), Miconia sp. (2,54\%), Caryocar brasiliense Cambess. (2,40\%), Xylopia frutescens Aubl. (2,14\%) e Eugenia dysenterica (Mart.) DC. (2,09\%).

Em relação à abundância, as espécies que se destacaram com os maiores índices foram: Qualea grandiflora Mart., com 271 indivíduos, Eriotheca pubescens (Mart. \& Zucc.) Schott \& Endl., 178 indivíduos, Magonia pubescens A.St.-Hil, 141 indivíduos, Copaifera langsdorffii Desf., 132 indivíduos, Machaerium opacum Vogel, 109 indivíduos, Miconia sp., 104 indivíduos (Tabela 1).

TABELA 1 - Parâmetros fitossociológicos para espécies amostradas no Cerrado sensu stricto de 1 ha, no Parque Estadual da Lapa Grande, Montes Claros- MG em ordem alfabética de famílias, onde: $\mathrm{N}$ - número de indivíduos, DR - densidade relativa, FR - frequência relativa, DoR dominância relativa, VI - valor de importância, PSR- posição sociológica relativo e VIA- valor de importância ampliado.

\begin{tabular}{|c|c|c|c|c|c|c|c|}
\hline Espécie & $\mathbf{N}$ & DR & FR & DoR & VI & PSR & VIA \\
\hline \multicolumn{8}{|l|}{ Anacardiaceae } \\
\hline Anacardium humile A.St.-Hil. & 2 & 0,07 & 0,29 & 0,24 & 0,20 & 0,05 & 0,25 \\
\hline Astronium fraxinifolium Schott & 42 & 1,37 & 1,17 & 2,12 & 1,55 & 1,28 & 2,83 \\
\hline Lithrea brasiliensis Marchand & 43 & 1,41 & 0,58 & 2,71 & 1,57 & 0,93 & 2,49 \\
\hline $\begin{array}{l}\text { Lithrea molleoides (Vell.) Engl. } \\
\text { Myracrodruon urundeuva }\end{array}$ & 43 & 1,41 & 0,58 & 1,43 & 1,14 & 0,98 & 2,12 \\
\hline Allemão & 36 & 1,18 & 0,73 & 1,66 & 1,19 & 0,66 & 1,85 \\
\hline Schinopsis brasiliensis Engl. & 2 & 0,07 & 0,29 & 0,04 & 0,13 & 0,08 & 0,21 \\
\hline $\begin{array}{l}\text { Schinus terebinthifolius Raddi } \\
\text { Annonaceae }\end{array}$ & 1 & 0,03 & 0,15 & 0,01 & 0,06 & 0,04 & 0,10 \\
\hline $\begin{array}{l}\text { Annona crassiflora Mart. } \\
\text { Annona emarginata (Schltdl.) }\end{array}$ & 2 & 0,07 & 0,29 & 0,34 & 0,23 & 0,08 & 0,31 \\
\hline $\begin{array}{l}\text { H.Rainer } \\
\text { Annona leptopetala (R.E.Fr.) }\end{array}$ & 9 & 0,29 & 0,44 & 0,1 & 0,28 & 0,36 & 0,63 \\
\hline H.Rainer & 2 & 0,07 & 0,15 & 0,02 & 0,08 & 0,05 & 0,13 \\
\hline Xylopia aromatica (Lam.) Mart. & 9 & 0,29 & 0,58 & 0,2 & 0,36 & 2,96 & 3,32 \\
\hline Xylopia frutescens Aubl. & 79 & 2,58 & 1,9 & 1,94 & 2,14 & 0,13 & 2,27 \\
\hline \multicolumn{8}{|l|}{ Apocynaceae } \\
\hline Aspidosperm & & & & & & & \\
\hline $\begin{array}{l}\text { Blake } \\
\text { NCICLOPÉDIA BIOSFERA. Cent }\end{array}$ & 1 & 0,03 & 0,15 & 0,03 & 0,07 & 0,04 & $\begin{array}{r}0,11 \\
2016\end{array}$ \\
\hline
\end{tabular}


Aspidosperma macrocarpon

Mart.

Aspidosperma pyrifolium Mart.

Aspidosperma sp

Aspidosperma subincanum Mart.

Aspidosperma tomentosum Mart.

Hancornia speciosa Gomes

Himatanthus obovatus (Müll.

Arg.) Woodson

$\begin{array}{rrrrrrr}3 & 0,1 & 0,29 & 0,03 & 0,14 & 0,12 & 0,26 \\ 63 & 2,06 & 1,75 & 1,21 & 1,67 & 2,07 & 3,74 \\ 1 & 0,03 & 0,15 & 0,01 & 0,06 & 0,04 & 0,10 \\ 4 & 0,13 & 0,44 & 0,12 & 0,23 & 0,13 & 0,36 \\ 54 & 1,77 & 1,9 & 0,89 & 1,52 & 2,09 & 3,61 \\ 6 & 0,2 & 0,58 & 0,22 & 0,33 & 0,24 & 0,57 \\ 2 & 0,07 & 0,29 & 0,04 & 0,13 & 0,05 & 0,19\end{array}$

Araliaceae

Schefflera macrocarpa (Cham. \&

Schltdl.) Frodin

$19 \quad 0,62 \quad 1,17 \quad 0,59 \quad 0,79 \quad 0,59 \quad 1,39$

Asteraceae

Chromolaena laevigata (Lam.)

R.M.King \& H.Rob.

Eremanthus elaeagnus (Mart. ex

DC.) Sch.Bip.

Eremanthus erythropappus (DC.)

MacLeish

Eremanthus glomerulatus Less.

Eremanthus incanus (Less.)

Less.

Eremanthus sp.

Piptocarpha rotundifolia (Less.)

Baker

Vernonia sp.

$\begin{array}{lllllll}3 & 0,1 & 0,29 & 0,05 & 0,15 & 0,12 & 0,27\end{array}$

$\begin{array}{lllllll}3 & 0,1 & 0,29 & 0,08 & 0,16 & 0,12 & 0,28\end{array}$

$\begin{array}{lllllll}3 & 0,1 & 0,29 & 0,09 & 0,16 & 0,07 & 0,23\end{array}$

$\begin{array}{lllllll}1 & 0,03 & 0,15 & 0,02 & 0,07 & 0,04 & 0,11\end{array}$

$\begin{array}{lllllll}2 & 0,07 & 0,29 & 0,04 & 0,13 & 0,05 & 0,19\end{array}$

$\begin{array}{lllllll}1 & 0,03 & 0,15 & 0,01 & 0,06 & 0,04 & 0,10\end{array}$

$\begin{array}{lllllll}40 & 1,31 & 1,46 & 1,03 & 1,26 & 1,43 & 2,69\end{array}$

$\begin{array}{lllllll}1 & 0,03 & 0,15 & 0,02 & 0,07 & 1,83 & 1,89\end{array}$

Bignoniaceae

Cybistax antisyphilitica (Mart.)

Mart.

Handroanthus chrysotrichus

(Mart. ex DC.) Mattos

Handroanthus ochraceus

(Cham.) Mattos

Handroanthus serratifolius (Vahl)

S.Grose

Tabebuia aurea (Silva Manso)

Benth.\& Hook.f. ex S.Moore

Tabebuia roseoalba (Ridl.)

Sandwith

$\begin{array}{lllllll}1 & 0,03 & 0,15 & 0,02 & 0,07 & 0,04 & 0,11\end{array}$

Zeyheria montana Mart.

$\begin{array}{lllllll}9 & 0,29 & 0,58 & 0,17 & 0,35 & 0,33 & 0,68\end{array}$

$\begin{array}{lllllll}7 & 0,23 & 0,73 & 0,1 & 0,35 & 0,28 & 0,63\end{array}$

$\begin{array}{lllllll}4 & 0,13 & 0,29 & 0,08 & 0,17 & 0,16 & 0,33\end{array}$

$2 \quad 0,07 \quad 0,15 \quad 0,03 \quad 0,08 \quad 0,08 \quad 0,16$

$\begin{array}{lllllll}1 & 0,03 & 0,15 & 0,02 & 0,07 & 0,01 & 0,08\end{array}$

$\begin{array}{lllllll}3 & 0,1 & 0,44 & 0,21 & 0,25 & 0,08 & 0,33\end{array}$

\section{Boraginaceae}

Cordia trichotoma (Vell.) Arráb.

ex Steud.

$\begin{array}{lllllll}1 & 0,03 & 0,15 & 0,02 & 0,06 & 0,04 & 0,10\end{array}$

Calophyllaceae

Kielmeyera coriacea Mart. \&

\begin{tabular}{llllllll} 
Zucc. & 5 & 0,16 & 0,44 & 0,13 & 0,24 & 0,20 & 0,44 \\
\hline Kielmeyera speciosa A.St.-Hil. & 1 & 0,03 & 0,15 & 0,11 & 0,10 & 0,01 & 0,11
\end{tabular}

Caryocaraceae 


\begin{tabular}{|c|c|c|c|c|c|c|c|}
\hline $\begin{array}{l}\text { Caryocar brasiliense Cambess. } \\
\text { Celastraceae }\end{array}$ & 25 & 0,82 & 1,31 & 5,06 & 2,40 & 0,80 & 3,20 \\
\hline $\begin{array}{l}\text { Plenckia populnea Reissek } \\
\text { Chrysobalanaceae }\end{array}$ & 11 & 0,36 & 0,58 & 0,13 & 0,36 & 0,44 & 0,79 \\
\hline $\begin{array}{l}\text { Licania rigida Benth. } \\
\text { Clethraceae }\end{array}$ & 1 & 0,03 & 0,15 & 0,04 & 0,07 & 0,04 & 0,11 \\
\hline $\begin{array}{l}\text { Clethra scabra Pers. } \\
\text { Combretaceae }\end{array}$ & 1 & 0,03 & 0,15 & 0,05 & 0,08 & 0,01 & 0,09 \\
\hline $\begin{array}{l}\text { Buchenavia tomentosa Eichler } \\
\text { Combretum duarteanum }\end{array}$ & 1 & 0,03 & 0,15 & 0,04 & 0,07 & 0,04 & 0,11 \\
\hline Caml & 2 & 0,07 & 0,29 & 0,02 & 0,13 & 0,08 & 0,21 \\
\hline Terminalia & 18 & 0,59 & 1,31 & 0,89 & 0,93 & 0,47 & 1,40 \\
\hline Terminalia fagifolia Mart. & 45 & 1,47 & 1,17 & 3,06 & 1,90 & 1,38 & 3,28 \\
\hline $\begin{array}{l}\text { Terminalia glabrescens Mart. } \\
\text { Cunoniaceae }\end{array}$ & 12 & 0,39 & 0,73 & 0,34 & 0,49 & 0,37 & 0,86 \\
\hline $\begin{array}{l}\text { Lamanonia ternata Vell. } \\
\text { Dilleniaceae }\end{array}$ & 1 & 0,03 & 0,15 & 0,04 & 0,07 & 0,01 & 0,09 \\
\hline $\begin{array}{l}\text { Curatella am } \\
\text { Davilla rugo: }\end{array}$ & 10 & 0,33 & 0,73 & 0,69 & 0,58 & 0,25 & 0,83 \\
\hline$D a$ & 10 & 0,3 & 0,58 & 0,14 & 0,35 & 0,40 & 0,75 \\
\hline
\end{tabular}

Ebenaceae

Diospyros hispida A.DC. $\quad \begin{array}{lllllll}5 & 0,16 & 0,44 & 0,08 & 0,23 & 0,17 & 0,40\end{array}$

\section{Erythroxylaceae}

Erythroxylum argentinum

O.E.Schulz



Erythroxylum suberosum A.St.-

Hil.

$\begin{array}{lllllll}10 & 0,33 & 1,02 & 0,29 & 0,55 & 0,37 & 0,92\end{array}$

Erythroxylum tortuosum Mart.

$\begin{array}{lllllll}3 & 0,1 & 0,29 & 0,12 & 0,17 & 0,12 & 0,29\end{array}$

Euphorbiaceae

$\begin{array}{llllllll}\text { Maprounea guianensis Aubl. } & 50 & 1,63 & 0,29 & 0,72 & 0,88 & 1,74 & 2,62\end{array}$

Fabaceae

Albizia niopoides (Spruce ex

Benth.) Burkart

Anadenanthera colubrina (Vell.)

Brenan

Bowdichia virgilioides Kunth

Chamaecrista sp.

Copaifera langsdorffii Desf.

Dalbergia decipularis Rizzini \&

A.Mattos

Dalbergia miscolobium Benth.

Dalbergia villosa (Benth.) Benth.

Dimorphandra mollis Benth.

Enterolobium gummiferum (Mart.)

J.F.Macbr.

$\begin{array}{lllllll}3 & 0,1 & 0,15 & 0,43 & 0,23 & 0,09 & 0,32\end{array}$

$\begin{array}{llllllll}\text { Hymenaea stigonocarpa Mart. ex } & 85 & 2,78 & 2,04 & 4,44 & 3,09 & 2,55 & 5,64\end{array}$ Hayne 
Leptolobium dasycarpum Vogel

Machaerium opacum Vogel

Machaerium villosum Vogel

Mimosa laticifera Rizzini \&

A.Mattos

Peltophorum dubium (Spreng.)

Taub.

Plathymenia reticulata Benth.

Pterogyne nitens Tul.

Schwartzia adamantium

Stryphnodendron adstringens

(Mart.) Coville

Swartzia flaemingii Raddi

Swartzia langsdorffii Raddi

Swartzia macrostachya Benth.

Swartzia oblata R.S.Cowan

Swartzia sp.

Tachigali aurea Tul.

Tachigali sp

Vatairea macrocarpa (Benth.)

Ducke
(Cambess.)Bedell ex Gir.-Cañas

Zollernia ilicifolia (Brongn.) Vogel

$\begin{array}{rrrrlll}40 & 1,31 & 1,75 & 0,83 & 1,30 & 1,45 & 2,75 \\ 109 & 3,56 & 2,48 & 3,35 & 3,13 & 3,41 & 6,54 \\ 3 & 0,1 & 0,44 & 0,04 & 0,19 & 0,12 & 0,31 \\ & 0,1 & 0,15 & 0,08 & 0,11 & 0,12 & 0,23 \\ 3 & 0,1 & & & & & \\ 1 & 0,03 & 0,15 & 0,01 & 0,06 & 0,04 & 0,10 \\ 34 & 1,11 & 1,17 & 2,62 & 1,63 & 0,58 & 2,21 \\ 2 & 0,07 & 0,29 & 0,02 & 0,13 & 0,08 & 0,21 \\ & 0,03 & 0,15 & 0,02 & 0,07 & 0,04 & 0,11 \\ 1 & 0,03 & & & & & \\ 15 & 0,49 & 0,87 & 0,31 & 0,56 & 0,54 & 1,10 \\ 3 & 0,1 & 0,29 & 0,04 & 0,14 & 0,12 & 0,26 \\ 2 & 0,07 & 0,29 & 0,04 & 0,13 & 0,05 & 0,19 \\ 1 & 0,03 & 0,15 & 0,01 & 0,06 & 0,04 & 0,10 \\ 2 & 0,07 & 0,15 & 0,01 & 0,08 & 0,08 & 0,16 \\ 2 & 0,07 & 0,15 & 0,14 & 0,12 & 0,08 & 0,20 \\ 33 & 1,08 & 0,87 & 0,81 & 0,92 & 1,26 & 2,18 \\ 2 & 0,07 & 0,15 & 0,6 & 0,27 & 0,00 & 0,27 \\ & & & & & & \\ 6 & 0,2 & 0,29 & 0,16 & 0,22 & 0,04 & 0,26 \\ 2 & 0,07 & 0,29 & 0,03 & 0,13 & 0,00 & 0,13 \\ & & & & & & \\ 7 & 0,23 & 0,73 & 0,08 & 0,35 & 0,25 & 0,60\end{array}$

Aegiphila verticillata Vell.

Loganiaceae

Strychnos pseudoquina A.St.-Hil. $\quad \begin{array}{llllllll}11 & 0,36 & 0,58 & 0,23 & 0,39 & 0,38 & 0,78\end{array}$

Lythraceae

Lafoensia pacari A.St.-Hil.

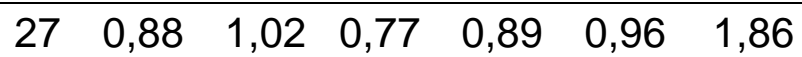

Malpighiaceae

Byrsonima sp.

20,07

$\begin{array}{llll}0,15 & 0,03 & 0,08 \\ 0,15 & 0,02 & 0,07 & 0\end{array}$

0,04

0,12

Byrsonima coccolobifolia Kunth

Byrsonima crassifolia (L.) Kunth

Byrsonima verbascifolia (L.) DC.

Heteropterys byrsonimifolia

A.Juss.

10,03

0,15

$\begin{array}{lll}0,02 & 0,07\end{array}$

0,08

0,15

10,03

0,15

$0,01 \quad 0,06$

0,04

0,10

$\begin{array}{lllllll}32 & 1,05 & 1,6 & 0,57 & 1,07 & 1,24 & 2,32\end{array}$

$\begin{array}{lllllll}51 & 1,67 & 1,31 & 1,29 & 1,42 & 1,72 & 3,14\end{array}$

Malvaceae

Eriotheca candolleana

(K.Schum.) A.Robyns

Eriotheca pentaphylla (Vell. \&

K.Schum.) A.Robyns

Eriotheca pubescens (Mart. \&

Zucc.) Schott \& Endl.

Guazuma ulmifolia Lam.

$1 \quad 0,03 \quad 0,15 \quad 0,01 \quad 0,06 \quad 0,04 \quad 0,10$

$\begin{array}{lllllll}4 & 0,13 & 0,44 & 0,21 & 0,26 & 0,10 & 0,36\end{array}$

$178 \quad 5,82 \quad 2,62 \quad 7,28 \quad 5,24 \quad 5,95 \quad 11,19$

$\begin{array}{lllllll}14 & 0,46 & 0,44 & 0,63 & 0,51 & 0,22 & 0,73\end{array}$

$\begin{array}{llllllll}\text { Luehea grandiflora Mart. \& Zucc. } \quad 28 & 0,92 & 0,58 & 1,2 & 0,90 & 0,53 & 1,43\end{array}$

Melastomataceae

Miconia albicans (Sw.) Triana

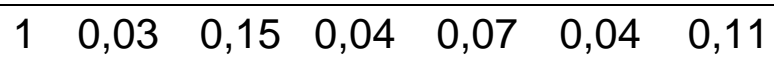




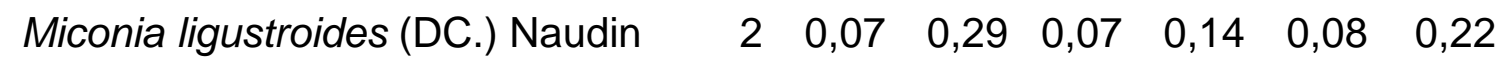
Miconia sp.

$\begin{array}{lllllll}104 & 3,4 & 1,31 & 2,91 & 2,54 & 4,02 & 6,56\end{array}$

Meliaceae

Guarea guidonia (L.) Sleumer $\quad 25 \quad 0,82 \quad 0,29 \quad 1,14 \quad 0,75 \quad 0,45 \quad 1,20$

Trichilia hirta L.

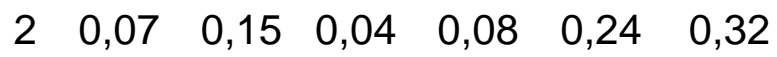

Moraceae

$\begin{array}{llllllll}\text { Brosimum gaudichaudii Trécul } \quad 26 & 0,85 & 0,87 & 0,28 & 0,67 & 0,87 & 1,54\end{array}$

Myristicaceae

$\begin{array}{llllllll}\text { Virola sebifera Aubl. } & 77 & 2,52 & 1,31 & 2,15 & 1,99 & 0,36 & 2,35\end{array}$

Myrtaceae

Calyptranthes concinna DC.

Campomanesia guazumifolia

(Cambess.) O.Berg

Eugenia acutata Miq.

Eugenia dysenterica (Mart.) DC.

Eugenia florida DC.

Eugenia speciosa Cambess.

Myrcia guianensis (Aubl.) DC.

Myrcia pulchra (O.Berg) Kiaersk.

Myrcia tomentosa (Aubl.) DC.

Myrciaria floribunda (H.West ex

Willd.) O.Berg

Myrciaria glanduliflora (Kiaersk.)

Mattos \& D.Legrand

Myrciaria guaniensis (H.West ex

Willd.) O.Berg

Plinia cauliflora (Mart.) Kausel

Psidium firmum O.Berg

Psidium myrsinites DC.

Psidium sartorianum (O.Berg)

Nied.

$\begin{array}{rrrrrrr}2 & 0,07 & 0,29 & 0,03 & 0,13 & 0,08 & 0,21 \\ 2 & 0,07 & 0,29 & 0,18 & 0,18 & 0,03 & 0,21 \\ 5 & 0,16 & 0,15 & 0,06 & 0,12 & 0,20 & 0,32 \\ 63 & 2,06 & 2,48 & 1,73 & 2,09 & 2,15 & 4,24 \\ 2 & 0,07 & 0,15 & 0,04 & 0,08 & 0,05 & 0,14 \\ 36 & 1,18 & 1,31 & 0,69 & 1,06 & 1,04 & 2,10 \\ 25 & 0,82 & 0,44 & 0,42 & 0,56 & 0,94 & 1,50 \\ 1 & 0,03 & 0,15 & 0,01 & 0,06 & 0,04 & 0,10 \\ 21 & 0,69 & 1,17 & 0,46 & 0,77 & 0,51 & 1,28 \\ & & & & & & \\ 21 & 0,69 & 0,58 & 0,26 & 0,51 & 0,81 & 1,32 \\ 1 & 0,03 & 0,15 & 0,02 & 0,06 & 0,01 & 0,08 \\ 1 & 0,03 & 0,15 & 0,02 & 0,07 & 0,04 & 0,11 \\ 37 & 1,21 & 0,73 & 0,55 & 0,83 & 1,39 & 2,22 \\ 1 & 0,03 & 0,15 & 0,01 & 0,06 & 0,04 & 0,10 \\ 6 & 0,2 & 0,15 & 0,18 & 0,18 & 0,16 & 0,33 \\ 4 & 0,13 & 0,15 & 0,03 & 0,10 & 0,16 & 0,26\end{array}$

Nyctaginaceae

Guapira graciliflora (Mart. ex

Schmidt) Lundell

Guapira noxia (Netto) Lundell

Guapira opposita (Vell.) Reitz

Guapira sp.

Neea theifera Oerst.

$\begin{array}{rrrrrrr}8 & 0,26 & 0,58 & 0,08 & 0,31 & 0,32 & 0,63 \\ 56 & 1,83 & 1,75 & 1,77 & 1,78 & 2,01 & 3,79 \\ 1 & 0,03 & 0,15 & 0,01 & 0,06 & 0,04 & 0,10 \\ 1 & 0,03 & 0,15 & 0,01 & 0,06 & 0,04 & 0,10 \\ 1 & 0,03 & 0,15 & 0,01 & 0,06 & 0,04 & 0,10\end{array}$

Ochnaceae

Ouratea hexasperma (A.St.-Hil.)

Baill.

$15 \quad 0,49 \quad 0,87 \quad 0,37 \quad 0,58 \quad 0,50 \quad 1,08$

Ouratea spectabilis (Mart.) Engl. $\quad \begin{array}{llllllll}1 & 0,03 & 0,15 & 0,03 & 0,07 & 0,04 & 0,11\end{array}$

Opiliaceae

Agonandra brasiliensis Miers ex

Benth. \& Hook.f.

$\begin{array}{lllllll}5 & 0,16 & 0,29 & 0,21 & 0,22 & 0,17 & 0,39\end{array}$

Peraceae

Pera sp

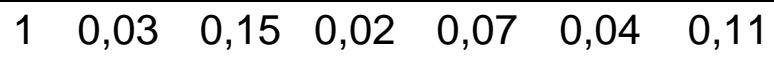

ENCICLOPÉDIA BIOSFERA, Centro Científico Conhecer - Goiânia, v.13 n.24; p.298 2016 
Polygonaceae

Coccoloba brasiliensis Nees \& Mart.

$15 \quad 0,49 \quad 0,29 \quad 0,26 \quad 0,35 \quad 0,60 \quad 0,94$

Coccoloba rosea Meisn.

$2 \quad 0,07 \quad 0,15 \quad 0,1 \quad 0,10 \quad 0,05 \quad 0,16$

Proteaceae

Roupala montana Aubl. $\quad \begin{array}{lllllll}10 & 0,33 & 0,73 & 0,16 & 0,40 & 0,40 & 0,80\end{array}$

Rubiaceae

$\begin{array}{llllllll}\text { Cordiera elliptica (Cham.) Kuntze } & 3 & 0,1 & 0,44 & 0,05 & 0,19 & 0,09 & 0,29\end{array}$

$\begin{array}{llllllll}\text { Cordiera sessilis (Vell.) Kuntze } \quad 36 & 1,18 & 1,31 & 0,99 & 1,16 & 1,32 & 2,48\end{array}$

Randia ferox (Cham. \& Schltdl.)

DC.

Tocoyena formosa (Cham. \&

Schltdl.) K.Schum.

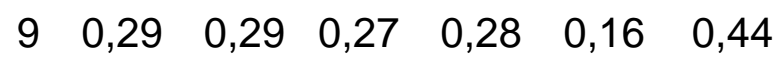

Rutaceae

Zanthoxylum rhoifolium Lam. $\quad \begin{array}{llllllll}8 & 0,26 & 0,58 & 0,68 & 0,51 & 0,05 & 0,56\end{array}$

Salicaceae

$\begin{array}{llllllll}\text { Casearia arborea (Rich.) Urb. } & 1 & 0,03 & 0,15 & 0,01 & 0,06 & 0,04 & 0,10\end{array}$

$\begin{array}{lllllllll}\text { Casearia rupestris Eichler } & 4 & 0,13 & 0,29 & 0,09 & 0,17 & 0,10 & 0,27\end{array}$

$\begin{array}{llllllll}\text { Casearia sylvestris Sw. } \quad 4 & 4 & 0,13 & 0,29 & 0,04 & 0,16 & 0,16 & 0,32\end{array}$

Sapindaceae

Dilodendron bipinnatum Radlk. $\quad \begin{array}{llllllll}2 & 0,07 & 0,15 & 0,13 & 0,11 & 0,03 & 0,14\end{array}$

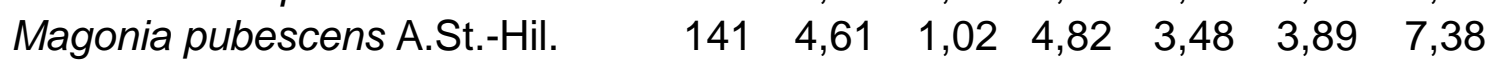

Sapotaceae

$\begin{array}{llllllll}\text { Chrysophyllum splendens } & 1 & 0,03 & 0,15 & 0,02 & 0,06 & 0,04 & 0,10\end{array}$

$\begin{array}{llllllll}\text { Sppengeria ramiflora (Mart.) Radlk. } \quad 26 & 0,85 & 1,46 & 0,79 & 1,03 & 0,92 & 1,96\end{array}$

Simaroubaceae

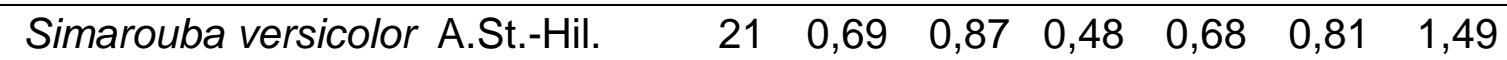

Solanaceae

$\begin{array}{llllllll}\text { Solanum sp. } & 1 & 0,03 & 0,15 & 0,03 & 0,07 & 0,01 & 0,08\end{array}$

Verbenaceae

Lantana sp.

Verbenaceae 1

$\begin{array}{lllllll}5 & 0,16 & 0,15 & 0,21 & 0,17 & 0,12 & 0,29\end{array}$

Vochysiaceae

Callisthene major Mart. \& Zucc.

Qualea grandiflora Mart.

Qualea multiflora Mart.

Qualea multiflora subsp.

Pubescens (Mart.) Stafleu

Qualea parviflora Mart.

Salvertia convallariodora A.St.-

Hil.

Vochysia elliptica Mart.

Vochysia thyrsoidea Pohl

Vochysia tucanorum Mart.

Sem família identificada

$\begin{array}{llllllll}1 & 0,03 & 0,15 & 0,03 & 0,07 & 0,04 & 0,11\end{array}$

Sp 1

$\begin{array}{rrrrrrr}2 & 0,07 & 0,29 & 0,05 & 0,13 & 0,08 & 0,21 \\ 271 & 8,86 & 3,06 & 9,01 & 6,98 & 9,73 & 16,71 \\ 46 & 1,5 & 1,9 & 0,92 & 1,44 & 1,61 & 3,05 \\ & & & & & & \\ 3 & 0,1 & 0,29 & 0,06 & 0,15 & 0,12 & 0,27 \\ 48 & 1,57 & 1,75 & 1,11 & 1,48 & 1,82 & 3,30 \\ & & & & & & \\ 2 & 0,07 & 0,15 & 0,32 & 0,18 & 0,05 & 0,23 \\ 9 & 0,29 & 0,44 & 0,1 & 0,28 & 0,10 & 0,38 \\ 6 & 0,2 & 0,44 & 1,36 & 0,66 & 0,04 & 0,70 \\ 1 & 0,03 & 0,15 & 0,26 & 0,15 & 0,25 & 0,40\end{array}$

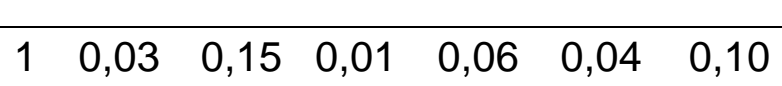




\begin{tabular}{lrrrrrrr} 
Sp 11 & 1 & 0,03 & 0,15 & 0,01 & 0,06 & 0,01 & 0,08 \\
Sp 12 & 1 & 0,03 & 0,15 & 0,01 & 0,06 & 0,01 & 0,08 \\
Sp 13 & 1 & 0,03 & 0,15 & 0,01 & 0,06 & 0,04 & 0,10 \\
Sp 15 & 1 & 0,03 & 0,15 & 0,02 & 0,07 & 0,01 & 0,08 \\
Sp 3 & 1 & 0,03 & 0,15 & 0,01 & 0,06 & 0,01 & 0,08 \\
Sp 4 & 1 & 0,03 & 0,15 & 0,01 & 0,06 & 0,04 & 0,10 \\
Sp 5 & 1 & 0,03 & 0,15 & 0,01 & 0,06 & 0,04 & 0,10 \\
Sp 6 & 1 & 0,03 & 0,15 & 0,01 & 0,06 & 0,04 & 0,10 \\
Sp 7 & 1 & 0,03 & 0,15 & 0,01 & 0,06 & 0,04 & 0,10 \\
Sp 8 & 1 & 0,03 & 0,15 & 0,02 & 0,07 & 0,04 & 0,11 \\
Sp 9 & 1 & 0,03 & 0,15 & 0,01 & 0,06 & 0,04 & 0,10 \\
\hline Total Geral & $\mathbf{3 0 5 9}$ & $\mathbf{1 0 0}$ & $\mathbf{1 0 0}$ & $\mathbf{1 0 0}$ & $\mathbf{1 0 0}$ & $\mathbf{1 0 0}$ & $\mathbf{2 0 0}$ \\
\hline
\end{tabular}

As espécies que foram destaque com maior IVI e densidade estiveram presentes na maioria das parcelas, como a Qualea grandiflora Mart. que exibiu uma melhor distribuição espacial, sendo encontrada em 21 de 25 unidades amostrais, seguida de Eriotheca pubescens (Mart. \& Zucc.) Schott \& Endl. em 18, Machaerium opacum Vogel e Eugenia dysenterica (Mart.) DC. em 17, Dimorphandra mollis Benth. em 16 e Hymenaea stigonocarpa Mart. ex Hayne em 14. Essas espécies são características do Cerrado sensu stricto e esse fato pode explicar seu amplo espectro de ocupação na área e sua importância para a dinâmica e conservação da biota local.

$\mathrm{O}$ índice de diversidade de Shannon ( $\left.\mathrm{H}^{\prime}\right)$, foi de 4,12 nats.indivíduo ${ }^{-1}$ e a equabilidade de Pielou de 0,8. Esse valor de diversidade está acima de valores encontrados em outros estudos em cerrado sensu stricto, que variaram entre 3,11 e 4,033, em áreas situadas em Tocantins, Minas Gerais, Mato Grosso (LIMA et al., 2012; GIÁCOMO et al., 2013; FINGER \& FINGER, 2015; SILVA-NETO et al., 2016), confirmando assim, uma alta diversidade da área.

As espécies que se destacaram com os quesitos de abundância, distribuição espacial, densidade, frequência, e IVI totalizaram 22 espécies apresentando frutificação e floração entre os meses de agosto e outubro e dispersão em sua maioria anemocórica. As espécies demonstraram um comportamento de frutificação durante todo o ano, com um declínio nos meses de março a junho, que é o período de seca na região e um aumento significativo a partir do mês agosto, tendo um declínio nos meses de fevereiro a abril, a floração também ocorre durante todo o ano e tem um aumento a partir de julho com uma queda a partir do mês de setembro.

Esse comportamento da floração tem sido descrito por outros autores, ocorrendo com uma frequência durante todo ano, e nos períodos secos ou de transição para chuvoso, aumenta a ocorrência desse evento (LENZA \& KLINK, 2006). Em relação à frutificação, também tem sido observado a seu vínculo com as chuvas, pois em períodos secos poucos indivíduos têm apresentado essa fenofase (PILON et al., 2015). Esses quesitos irão proporcionar a decisão acertada de plantio, melhor época de coleta de sementes e frutos, propagação e dispersão de espécies, além de embasar análises de ameaças da vegetação (Figura 2). 


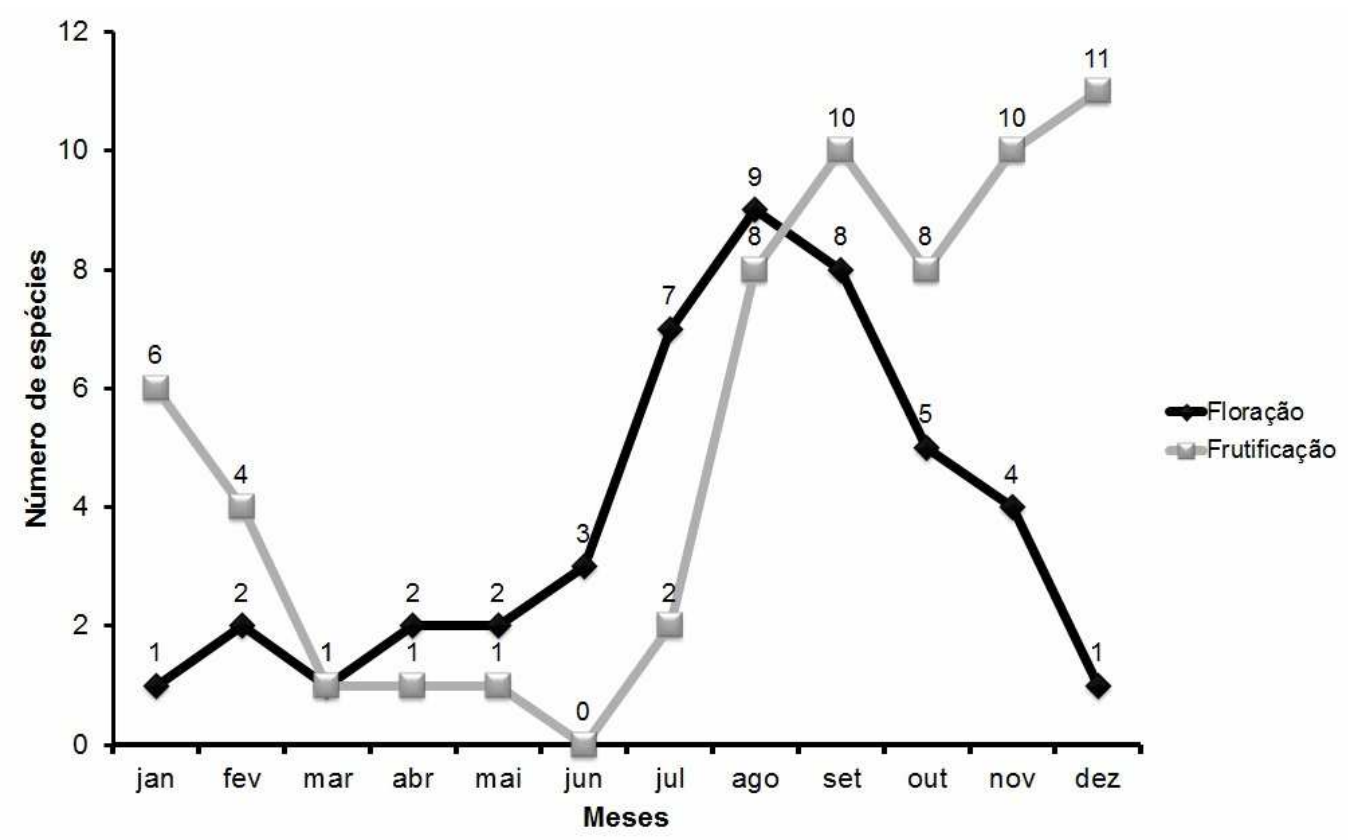

FIGURA 2 - Floração e Frutificação ao longo do ano das espécies encontradas. Fonte: Autores

Em relação à síndrome de dispersão, as espécies listadas apresentaram predominantemente característica de anemocóricas, com 14 espécies (66,67\%), sendo que os seus diásporos são dispersos pelo vento. As espécies que os diásporos são dispersos por animais, zoocóricas, totalizaram seis espécies $(28,57 \%)$, e as que são dispersos por gravidade ou explosão, autocóricas, com 1 espécie $(4,76 \%)$ (Figura 2).



FIGURA 3 - Síndrome de dispersão das espécies encontradas.

Fonte: Autores

Em áreas abertas como o cerrado sensu stricto, as espécies anemocóricas e autocóricas tem uma maior frutificação em períodos secos, devido a sua facilidade 
de dispersão (BATALHA et al., 1997). O levantamento apresentou uma elevada riqueza, com espécies que caracteriza de forma satisfatória o Cerrado sensu stricto. Além disso, os valores de diversidade pelo Índice de Shannou ressaltam a potencialidade de outros estudos em áreas de PELG e a importância de conservação desses recursos em uma região que sofre com déficit hídrico.

As espécies destacadas merecem um maior aprofundamento sobre suas características ecológicas, sivilculturais e sua função adaptativa nessas áreas, ademais podem ser utilizadas em arranjos de manejo da vegetação e rápida recomposição de áreas. O comportamento de floração e frutificação apresentou uma relação inerente com a sazonalidade climática, o que deve ser considerado nessa região, que apresenta muita variação de precipitação e clima.

\section{CONCLUSÃO}

O estudo apresentou uma riqueza de 171 espécies, distribuídas em 46 famílias, com as espécies Qualea grandiflora Mart., Eriotheca pubescens (Mart. \& Zucc.) Schott \& Endl e Magonia pubescens A.St.-Hil. com maiores valores de IVI .

A área de estudo possui uma alta diversidade no Cerrado do interior do PELG, quando comparado a outros estudos na mesma vegetação. As quatro famílias mais representativas foram Fabaceae, Vochysiaceae, Myrtaceae e Malvaceae.

As espécies mais representativas da vegetação do Cerrado sensu stricto do PELG somaram 22 espécies e mostrou um comportamento de fenologia com um aumento significativo de frutos e sementes a partir do mês de agosto e floração no mês de julho, e com a síndrome de dispersão predominantemente anemocórica, com mais de $60 \%$ das espécies observadas.

\section{REFERÊNCIAS}

ALMEIDA, R. F.; FAGG, C. W.; OLIVEIRA, M. C.; MUNHOZ, C. B. R.; LIMA, A. S.; OLIVEIRA, L. S. B. Mudanças florísticas e estruturais no cerrado sensu stricto ao longo de 27 anos (1985-2012) na Fazenda Água Limpa, Brasília, DF. Rodriguésia, v. $65, \quad$ n. $1, \quad 2014$. Disponível em<http://dx.doi.org/10.1590/S217578602014000100001>. Doi: 10.1590/S2175-78602014000100001.

BATALHA, M. A.; ARAGAKI, S.; MANTOVANI, W. Variações fenológicas das espécies do cerrado em emas (Pirassununga, SP). Acta Botanica Brasilica, v. 11, n. 1 , p. 61-78, 1997. Disponível em< http://dx.doi.org/10.1590/S010233061997000100007>. Doi: 10.1590/S0102-33061997000100007.

BRASIL. Guia de Campo. Vegetação do Cerrado 500 espécies. $2^{a}$ ed. Brasília DF: MMA/SFB. 2011.

BRASIL. Ministério do Meio Ambiente. Cerrado e Pantanal áreas e ações prioritárias para conservação da biodiversidade. Brasília, DF, 2007, 13p. Disponível

em<

http://www.mma.gov.br/estruturas/chm/_arquivos/cerrado_pantanal.pdf>. Acesso em: 22 set. 2016.

FERREIRA, A. C. A.; SILVA, K. A.; OLIVEIRA, N. C.; SILVA, P. M.; MARTINS, T. O. Composição florística, fitossociológica e similaridade de um fragmento de Floresta ENCICLOPÉDIA BIOSFERA, Centro Científico Conhecer - Goiânia, v.13 n.24; p.302 
Estacional Semidecidua no Cerrado. Revista Tree Dimensional, v. 1, n. 2, p. 23-33, 2016.

FINGER, Z.; FINGER, F. A. Fitossociologia em comunidades arbóreas remanescentes de Cerrado sensu stricto no Brasil Central. Revista Floresta, v. 45, n. 4, p. 769-780, 2015. Disponível em< http://dx.doi.org/10.5380/rf.v45i4.30860>. Doi: $10.5380 /$ rf.v45i4.30860.

FLORA DO BRASIL 2020 em construção. Jardim Botânico do Rio de Janeiro. Disponível em: < http://floradobrasil.jbrj.gov.br/ >. Acesso em: 23 Out. 2016.

GIÁCOMO, R. G.; CARVALHO, D. C.; PEREIRA, M. G.; SOUZA, A. B.; GAUI, T. D. Florística e Fitossociologia em áreas de campo sujo e Cerrado sensu stricto na Estação Ecológica De Pirapitinga - MG. Ciência Florestal, v. 23, n. 1, p. 29-43, 2013. Disponível em< http://dx.doi.org/10.5902/198050988437>. Doi: $10.5902 / 198050988437$.

IBGE. Manual Técnico da Vegetação Brasileira. $2^{\mathrm{a}}$ ed. Rio de Janeiro - RJ: Geociências. 2012.

JACOBI, C. M.; CARMO, F. F.; VINCENT, R. C. Estudo Fitossociológico de uma comunidade vegetal sobre canga como subsidio para a reabilitação de áreas mineradas no Quadrilátero Ferrífero, MG. Revista Árvore, v. 32, n. 2, p. 345-353, 2008. Disponível em< http://dx.doi.org/10.1590/S0100-67622008000200017>. Doi: $10.1590 /$ S0100-67622008000200017.

JBRJ - Instituto de Pesquisas Jardim Botânico do Rio de Janeiro. Jabot - Banco de Dados da Flora Brasileira. Disponível em: <http://www.jbrj.gov.br/jabot>. Acesso em: 23 Out. 2016.

LENZA, E.; KLINK, C. A. Comportamento fenológico de espécies lenhosas em um cerrado sentido restrito de Brasília, DF. Brazilian Journal of Botany, v. 29, n. 4, p. 627-638, 2006. Disponível em< http://dx.doi.org/10.1590/S010084042006000400013>. Doi: 10.1590/S0100-84042006000400013.

LIMA, I. L. P.; SCARIOT, A.; MEDEIROS, M. B.; SEVILHA, A. C. Diversidade e uso de plantas do Cerrado em comunidades de Geraizeiros no norte do estado de Minas Gerais, Brasil. Acta Botanica Brasilica, v. 26, n. 3, p. 675-684, 2012. Disponível em< http://dx.doi.org/10.1590/S0102-33062012000300017>. Doi: 10.1590/S010233062012000300017.

MAGURRAN, A. E. Medindo a diversidade biológica. Curitiba: Editora UFPR. 2011.

MINAS GERAIS (ESTADO). Decreto № 44.204, de 10 de janeiro de 2006. Cria o Parque Estadual da Lapa Grande, no Município de Montes Claros. Diário do Executivo, Minas Gerais, 11 jan. 2006. Disponível em< http://www.siam.mg.gov.br/sla/download.pdf?idNorma=5310>. Acesso em: 25 set.2016. 
MINAS GERAIS (ESTADO). Secretaria de Estado de Meio Ambiente e Desenvolvimento Sustentável. Plano Emergencial de uso público Parque Estadual da Lapa Grande. Montes Claros, MG, 2015. 10p.

MORO, M. F.; MARTINS, F. R. Métodos de levantamento do componente arbóreoarbustivo. In: FELFILI, J. M.; EISENLOHR, P. V.; MELO, M. M. R. F.; ANDRADE, L. A.; MEIRA NETO, J. A. A. (Org.). Fitossociologia no Brasil: Métodos e Estudos de Caso - v. 1. Viçosa: Editora UFV, 2011. p. 174-212.

MULLER-DOMBOIS, D.; ELLENBERG, H. Aims and methods of vegetation ecology. New York: John Wiley \& Sons. 1974.

OLIVEIRA, A. N.; AMARAL, I. L. Florística e fitossociologia de uma floresta de vertente na Amazônia Central, Amazonas, Brasil. Acta Amazonica, v. 34, n. 1, p. 21-34, 2004. Disponível em< http://dx.doi.org/10.1590/S0044-59672004000100004>. Doi: 10.1590/S0044-59672004000100004.

OLIVEIRA, P. S.; MOREIRA, A. A.; NERY, C. V. M.; MELO, A. A. M. Microcorredores Ecológicos no entorno do Parque Estadual da Lapa Grande. Caminhos da Geografia, Uberlândia, v. 16, n. 53, p. 189-200, 2015. Disponível em< http://www.seer.ufu.br/index.php/caminhosdegeografia/article/view/25118/16443>.

Acesso em: 19 set. 2016.

PILON, N. A. L.; UDULUTSCH, R. G.; DURIGAN, G. Padrões fenológicos de 111 espécies de Cerrado em condições de cultivo. Hoehnea, v. 42, n. 3, p. 425-443, 2015. Disponível em<http://dx.doi.org/10.1590/2236-8906-07/2015>. Doi: 10.1590/2236-8906-07/2015.

RIBEIRO, J. F.; WALTER, B. M. T. Fitofisionomias do Bioma Cerrado. In: SANO, S. M.; ALMEIDA, S. P. (Org.). Cerrado: ambiente e flora. Planaltina: Embrapa, 1998. p. 89-166.

SILVA JÚNIOR, M. C. 100 árvores do Cerrado Sentido Restrito. $1^{1} \underline{a}$ ed. Brasília DF: Rede de sementes do Cerrado. 2012.

SILVA JÚNIOR, M. C.; PEREIRA, B. A. S. 100 árvores do Cerrado - Matas de Galeria. $1^{\underline{a}}$ ed. Brasília - DF: Rede de sementes do Cerrado. 2009.

SILVA-NETO, V. L.; OLIVEIRA, A. L.; FERREIRA, R. Q. S.; SOUZA, P. B.; VIOLA, M. R. Fitossociologia e distribuição diamétrica de uma área de Cerrado sensu stricto, Dueré-TO. Revista de Ciências Ambientais, v. 10, n. 1, p. 91-106, 2016. Disponível em< http://dx.doi.org/10.18316/1981-8858.16.24>. Doi: 10.18316/19818858.16.24.

SOARES, M. P.; NUNES, Y. R. F. Regeneração natural de cerrado sob plantio de Eucalyptus camaldulensis Dehn. no norte de Minas Gerais, Brasil. Revista Ceres, v. 60, n. 2, p. 205-214, 2013. Disponível em< http://dx.doi.org/10.1590/S0034737X2013000200008>. Doi: 10.1590/S0034-737X2013000200008. 
SOUZA, A. L.; SOARES, C. P. B. Florestas Nativas - Estrutura, dinâmica e manejo. $1^{1}$ ed. Viçosa: Editora da Universidade Federal de Viçosa. 2013.

VAN DER PIJL, L. Principles of dispersal in higher plants. $3^{\text {a }}$ ed. Berlin: SpringerVerlag. 1982. 